\section{TumorBiology}

\author{
Shirley McBride \\ Paula Meleady: \\ Alan Baird ${ }^{b}$ \\ David Dinsdalec \\ Mantin $l y n e s$ a \\ a National Cell and Tissue Culture \\ Centre, BioResearch Ireland, \\ Dublin City University, and \\ b Pharmacology Department, \\ University College, \\ Dublin, Ireland; \\ c MRC Toxicology Unit, \\ University of Leicester, UK
}

\title{
Human Lung Carcinoma Cell Line DLKP Contains 3 Distinct Subpopulations with Different Growth and Attachment Properties
}

\begin{abstract}
Many human solid tumours, particularly lung tumours, contain different subpopulations, the presence of which can complicate diagnosis and treatment, yet few models exist for in vitro studies. We have found that DLKP, a human lung cell line established from a tumour histologically diagnosed as a 'poorly differentiated squamous carcinoma', contains 3 morphologically distinct populations. Three clones corresponding to these populations were established from the parental DLKP cells. Confirmation that the clones were derived from the parental population was obtained by DNA fingerprinting. The clones were designated $\mathrm{M}$ (mesenchymallike), I (intermediate) and SQ (squamous). On prolonged subculture, SQ and M can each interconvert with I, but SQ and $\mathrm{M}$ do not interconvert. We investigated the growth patterns of these isolated populations in monolayer culture, soft agar, spinner flasks and serum-free medium. In all but the latter assay, the parental DLKP cells grew faster than each of the clones, indicating some form of physiological co-operation between the clones. The growth of the clones themselves varied under the different assay conditions (DLKP-I showing greatest growth in monolayer, in serum-containing and serum-free media but, surprisingly, being unable to grow in soft agar, unlike the SQ and $\mathrm{M}$ clones). Addition of fibronectin permitted growth of DLKP-M and DLKP-SQ in serum-free medium at equivalent rates to those of DLKP and DLKP-I. In some cases, morphological adaptation to specific growth conditions was observed. Variation between the clones was also evident in their respective chromosome numbers (with the $\mathbf{M}$ clone being predominantly hyperdiploid and the other clones predominantly hypertetraploid) and in their ability to adhere to extracellular matrix proteins, with DLKP-M showing most rapid attachment. Electrical resistance studies revealed the absence of tight junctions from the parental line and clonal subpopulations. Extensive immunohistological studies showed that neither DLKP nor the clones express cytokeratin or any other epithelial marker examined, but neuroendocrine markers were present. Further analysis of these different clonal populations may help to reveal some of the mechanisms involved in lung tumour development and progression.
\end{abstract}

\section{Key Words}

Lung

Small-cell lung carcinoma

Clones

Heterogeneity

\begin{tabular}{ll}
\hline KARGER & $\oplus 1998$ S. Karger AG, Basel \\
Fax +41 61306 12 34 & 1010-4283/98/0192-0088 $\$ 15.00 / 0$ \\
$\begin{array}{l}\text { E-Mail karger@karger.ch } \\
\text { www.karger.com }\end{array}$ & $\begin{array}{l}\text { This article is also accessible online at: } \\
\text { http://BioMedNet.com/karger }\end{array}$
\end{tabular}

www.karger.com http://BioMedNet.com/karger
Dr. Shirley McBride

Department of Pathology, University of Edinburgh

Medical School

Teviot Place

Edinburgh EH8 9AG (Scotland) 


\section{Introduction}

Knowledge of the behaviour of heterogeneous subpopulations in solid tumours and in cell lines is crucial to our understanding of tumour development and progression. Heterogeneity is exhibited in many aspects of tumour biology including cellular morphology [1], karyotype [2, 3], growth rate [4], metastatic potential [5] and antigen expression [6], and lung cancers are particularly noted for their heterogeneity [7]. The normal lung itself is a complex organ of about 40 cell types, capable of rapid cellular proliferation, differentiation and tissue remodelling if required, and the intricate micro-environment necessary to control this undoubtedly contributes to the variation seen within individual lung tumours. The inherent multipotential nature of lung stem cells [8] also indicates that a loss of control of growth or differentiation could result in heterogeneous populations of cells.

While an accurate diagnosis of tumour type is important for choosing an appropriate treatment regimen, the diverse multidifferentiated state of many lung cancers makes this prediction difficult. Therapeutically, lung cancers are broadly classed as small cell lung carcinoma (SCLC) or non-small cell lung carcinoma (NSCLC) [9]. SCLC (30\% of lung cancers) exhibit neuroendocrine (NE) differentiation, with the expression of markers including $L$-dopa decarboxylase [10], creatine kinase$\mathrm{BB}$ isoenzyme [11], bombesin [12] and neuron-specific enolase (NSE) [13] immunoreactivity and dense core granules [14]. They also grow rapidly, metastasise early and respond relatively well, at least initially, to radiation treatment and chemotherapy. NSCLC (70\% of lung cancers) include squamous, adenocarcinomas and large cell carcinomas. They are generally radio- and chemoresistant and are treated by surgical resection. However, these broad categories are often inappropriate and misleading. Many variant SCLC (SCLC-V) cell lines have now been described $[15,16]$. These cells express some, but not all, of the characteristics associated with SCLC NE differentiation. Loss of these markers can be accompanied by increased radiation and chemotherapy resistance [15] similar to that seen in NSCLC. Likewise, NE features once believed to be exclusive to SCLC have since been demonstrated in many NSCLC tumours and cell lines [17, 18], now described as NSCLC-NE, where 2 or more NE markers are observed. Evidence that NSCLC-NE has a greater metastatic potential [19] and is more sensitive to radiation treatment and chemotherapy than NSCLC $[20,21]$ could influence the treatment of patients with such tumours.

The presence of overlapping features in SCLC and NSCLC reinforces the 'common stem cell' theory of lung cancer [22]. This states that all lung cancers arise from a common precursor cell in the epithelium. Indeed, in normal foetal lung development, all bronchial epithelial cells are derived from a single cell type which lines the tracheobronchial tree. Accepting this theory, along with the supposition that most cancers are monoclonal in origin, means that heterogeneity in tumours arises as a consequence of the physiological micro-environment already present in the lung along with tumour-specific mechanisms such as genetic instability, rather than multiple cell types giving rise to diverse tumour types. Therefore, examining the characteristics and behaviour of tumour subpopulations in isolation and in situ gives insight into tumour development, progression, heterogeneity and metastasis, as well as normal cellular differentiation. In this study, we have examined under various conditions the growth of 3 clones isolated from a lung cell line, with a view to understanding the significance of heterogeneous tumour subpopulations. 


\section{Materials and Methods}

\section{Cell Culture}

The cell line DLKP was established as described previously [23] from a lymph node metastasis of a primary lung tumour which was histologically diagnosed as a 'poorly differentiated squamous cell carcinoma'. The cells were cultured routinely in DMEM:Hams F12 (1:1) (Gibco BRL, Paisley, UK) supplemented with 5\% foetal calf serum (Sigma, Poole, UK) and $2 \mathrm{~m} M L$-glutamine (Gibco BRL) and were monitored regularly for mycoplasma contamination (carried out in our lab) While DLKP cells have been passaged over 90 times, for the experiments described here, only cells at passage numbers $20-35$ were used, unless otherwise stated.

\section{Establishment of DLKP Clones}

Clones of the DLKP cell line were obtained by a limiting dilution assay. An exponentially growing culture of DLKP cells (passage 5) was harvested by trypsinisation, diluted in growth medium and plated at a density of 1 cell per 3 wells of a 96-well cell culture plate (Falcon, Oxford, UK). The plates were incubated at $37^{\circ} \mathrm{C}$ in $5 \% \mathrm{CO}_{2}$ for 5 days, after which time wells containing 1 colony of cells only were marked and cultured further. The resulting clones were then re-cloned in the same manner to ensure true clonal populations. DLKP-I (intermediate clone) and DLKP-M (mesenchymal-like clone) were established in this manner. DLKP-SQ (squamous clone) was cloned once only.

\section{Determination of Chromosome Numbers}

Preparation and harvesting of metaphase cells for chromosome counting were carried out as described previously [23]. Chromosome numbers were determined by microscopic analysis. At least 50 metaphase spreads from each cell line were counted.

\section{Growth Assays}

Exponentially growing cells were used to set up all growth assays. To examine growth in monolayer culture, cells were plated in 96-well culture plates at densities of $1 \times 10^{3}$ cells $/ 100 \mu 1 /$ well in normal growth medium supplemented with $5 \%$ serum. Following overnight attachment at $37^{\circ} \mathrm{C}$ in $5 \% \mathrm{CO}_{2}$, cells were rinsed in pre-warmed PBS, and $100 \mu \mathrm{l}$ of fresh medium containing 0,1 or $5 \%$ serum was plated. At selected time points, the extent of growth was determined by a crystal violet dye elution assay [24].

In soft-agar assays, $1.5 \times 10^{4}$ cells suspended in $1.5 \mathrm{ml}$ of $0.3 \%$ agar in growth medium were layered on $1.5 \mathrm{ml}$ of $0.6 \%$ pre-set agar in growth medium in 35 $\mathrm{mm}$ tissue culture dishes (Falcon). Triplicate plates were incubated at $37^{\circ} \mathrm{C}$ in $5 \% \mathrm{CO}_{2}$ for 10 days. Colonies greater than $20 \mu \mathrm{m}$ in diameter were counted microscopically, and average colony-forming efficiency (CFE) was determined by expressing the number of colonies formed as a percentage of the number of cells plated.

Growth of the cells in suspension was examined using glass spinner flasks (Techne, Cambridge, UK) precoated with a siliconising agent, dimethyldichlorosilane solution (BDH, Poole, UK) to prevent cell attachment. Following sterilisation, flasks were inoculated with $4 \times 10^{4}$ cells $/ \mathrm{ml}$ in $50 \mathrm{ml}$ growth medium and incubated at $37^{\circ} \mathrm{C}$ on magnetic stirrers which rotated a rod $(35 \mathrm{rpm})$ inside the flasks, keeping the cells in suspension. After 7 days, cells were harvested and counted, and the flasks were re-seeded at initial inoculating densities. After 6 weeks in culture, cells were fed with $50 \mathrm{ml}$ growth medium 3-4 days after reseeding, and this was found to increase growth.

In the serum-free assays, the medium used was an adaptation of that described by Mendiaz et al. [25], containing $10 \mu \mathrm{g} / \mathrm{ml}$ insulin and $5 \mu \mathrm{g} / \mathrm{ml}$ transferrin (both from Sigma). Cells were cultured in $25-\mathrm{cm}^{2}$ vented flasks (Costar, Cambridge, Mass., USA), and proliferation was determined by cell counts.

\section{Attachment Assays}

$50 \mu \mathrm{g} / \mathrm{ml}$ extracellular matrix (ECM) proteins collagen type IV, laminin and fibronectin (all from Sigma) in PBS-A were allowed to ad sorb to 24-well plates (Falcon) overnight at $4^{\circ} \mathrm{C}$. Wells were rinsed with PBS and blocked with BSA $\left(0.1 \%\right.$; Sigma) $/ \mathrm{PBS}$ at $37^{\circ} \mathrm{C}$ for $15 \mathrm{~min}$. Wells were rinsed again, and cells were plated at $1 \times 10^{4}$ cells/well in DMEM:Hams F12 (1:1) without serum. Cells were allowed to attach at $37^{\circ} \mathrm{C}$, and at each time point, medium and unattached cells were removed, the wells were rinsed gently with PBS and attached cells were stained with crystal violet dye $(\mathrm{BDH})$. Cell attachment was determined by a crystal violet dye elution assay.

\section{Electrical-Resistance Studies}

Cell monolayers were seeded at confluent densities into porous inserts (Falcon), suspended in 12-well tissue culture plates (Falcon) and incubated at $37^{\circ} \mathrm{C}$ in $5 \% \mathrm{CO}_{2}$ for the duration of the assay. Electrical-resistance measurements were carried out using an Endohm apparatus (World Precision Instruments, Fla., USA) which registered the resistance presented by the monolayer to an electrical current. T84 colonic epithelial cells (ATCC, Rockville, Md., USA) were used as a positive control. 
Immunological-Marker Detection

For immunocytochemical analysis, cells were either grown on multiwell glass slides (CML, Nemours, France) or cytocentrifuged onto glass slides and fixed in ice-cold methanol $(7 \mathrm{~min})$ and acetone $(2 \mathrm{~min})$. A limited number of formalin-fixed paraffin-embedded sections of the original tumour were available for analysis. Staining was performed by the avidin-biotin-peroxidase technique using DAKO Labs ABC visualising kits (DAKO, High Wycombe, UK) according to the manufacturer's instructions. Primary antibodies used were as follows: anti-CAM 5.2 (Becton-Dickinson, Erembodegem, Belgium), pan-cytokeratin (Sigma), epithelial membrane antigen (DAKO), epithelial-specific antigen (Sigma), desmoplakin (gift from Dr. David Garrod, University of Manchester, Manchester, UK), desmosomal protein (Sigma), transglutaminase (Biogenesis, Poole, UK), $\alpha_{2}$-integrin (gift from Dr. Fiona Watt, Imperial Cancer Research Fund, London, UK), neuron-specific enolase (NSE; DAKO), protein gene product 9.5 (PGP 9.5; Ultraclone Cambridge, UK) Leu 7 (Becton-Dickinson), neural-cell adhesion molecule (DAKO), serotonin (DAKO), chromogranin (DAKO), neurofilaments (Immunotech, Marseille, France), glial fibrillary acidic protein (DAKO), vimentin (DAKO), desmin (Sigma) and lymphocyte common antigen (DAKO).

\section{Results}

\section{Establishment of Clones}

Three morphologically distinct clones, DLKP-SQ, DLKP-I and DLKP-M, were isolated from the parental DLKP population (fig. 1). DLKP-I and DLKP-M were cloned and then re-cloned, while DLKP-SQ was cloned once. The morphologies of clones DLKP-SQ and DLKP-I are apparent in DLKP, but the DLKP-M morphology appears slightly altered in isolation. Each clone has been DNA fingerprinted (CellMark, Oxford, UK; results not shown) and shown to be derived from DLKP.

The DLKP-SQ morphology appears to predominate in the parental DLKP population $(\sim 70 \%)$. These cells are relatively large squamous-like cells which form colonies with distinct cell boundaries in monolayer culture. The DLKP-I morphology accounts for 25\% of the DLKP population. These cells are smaller than DLKP-SQ and grow in more tightly packed colonies, with indistinct cell boundaries. DLKP-M appears to account for the remaining DLKP population $(\sim 5 \%)$. In isolation, these cells are of intermediate size, with irregular elongated shapes and possess short neurite-like processes. They do not form colonies in monolayer culture, but instead grow in a scattered manner, with some cells extending across neighbouring cells. In the mixed parental population, these cells have a more regular flattened appearance, but again, they do not form colonies. Interconversion occurs between the clones after about 20-25 passages in isolation. After this time, colonies with DLKP-I morphology begin to appear in cultures of DLKP-M and DLKP-SQ, and similarly, colonies with DLKP-SQ morphology, and to a lesser extent DLKP-M morphology, can be seen in cultures of DLKP-I (fig. 1b, 2). No interconversion has been observed between DLKP-M and DLKP-SQ.

\section{Determination of Chromosome Numbers}

Analysis of the respective chromosomal numbers of DLKP and its 3 clones revealed a range of chromosomal populations within each line, but prominent populations were evident in each case (fig. 3). The largest DLKP chromosomal population was hypertetraploid, with $40 \%$ of the cells containing 100-110 chromosomes. Thirty-six percent of DLKP-I cells were roughly tetraploid (90-100 chromosomes), and DLKP-M exhibited the closest-to-normal chromosome complement, with $60 \%$ of cells being hyperdiploid (50-60 chromosomes). DLKP-SQ cells had the most varied chromosome numbers. Thirty-six percent of the cells had modal numbers between 90 and 100 , while $27 \%$ had numbers between 100 and 110 . 


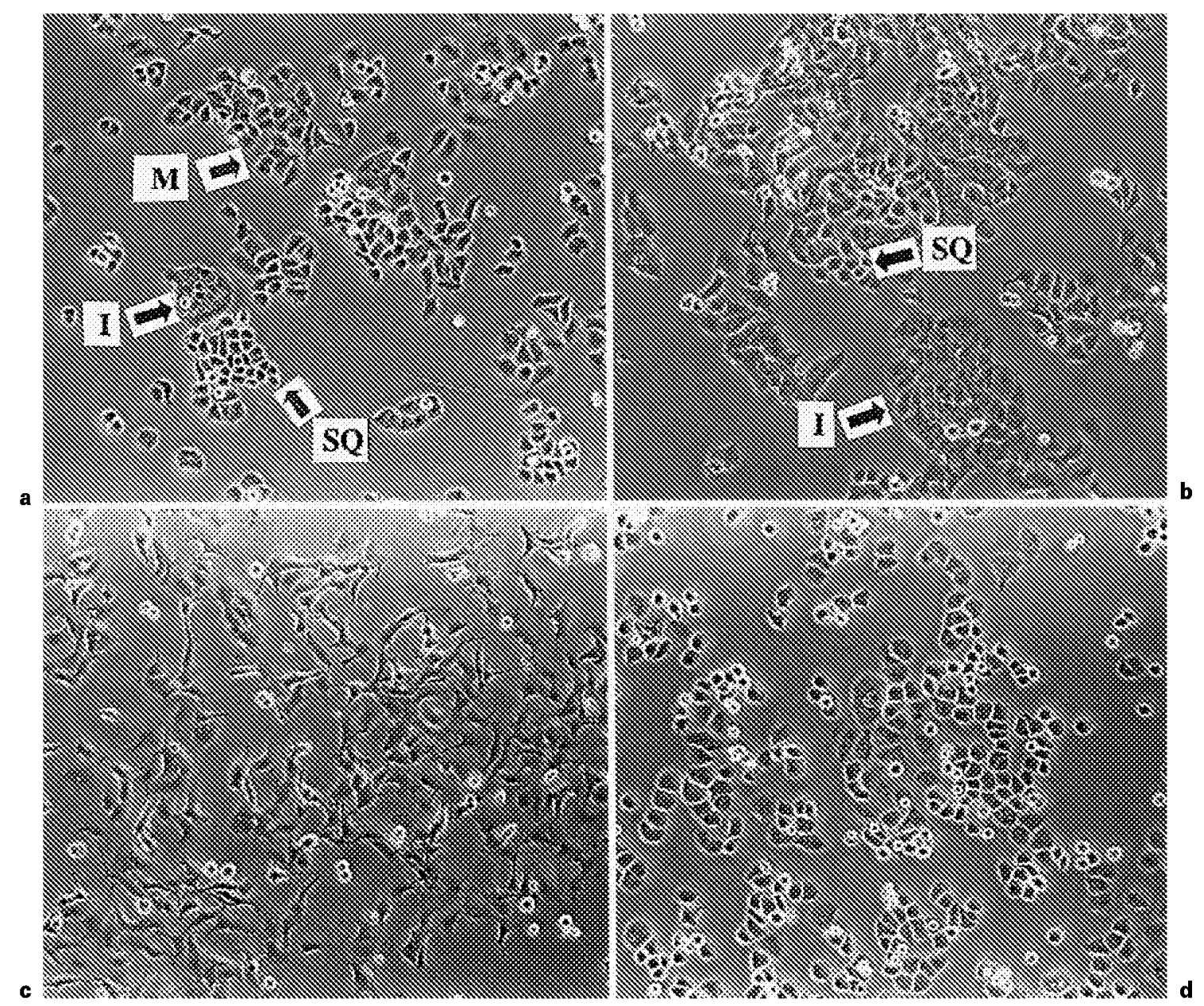

Fig. 1. Morphological appearance of DLKP (a), DLKP-I (b), DLKP-M (c) and DLKPSQ (d) cultured in DMEM:Hams F12 (1:1) supplemented with 5\% serum. Note that in b, DLKP-SQ-like morphologies begin to appear in cultures of DLKP-I. I = DLKP-I like; $\mathbf{M}=$ DLKP-M like; SQ = DLKP-SQ like. $\times 100$.

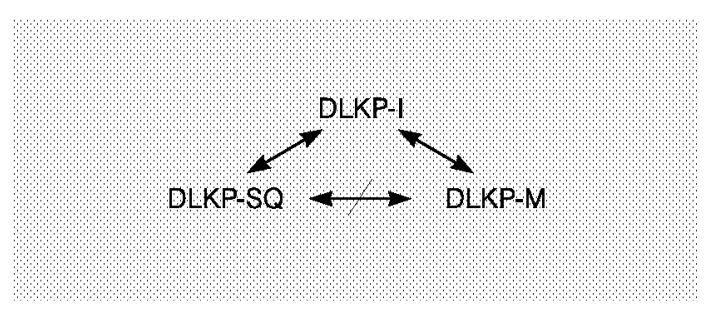

Fig. 2. Apparent interconversion of DLKP clones observed in culture.

\section{Growth of DLKP and Clones}

The growth curves of DLKP and its 3 clones in monolayer culture were determined in the presence of 0,1 and $5 \%$ serum (fig. 4). At each serum concentration, the parental DLKP cells proliferated faster than any of the clones. Of the clones themselves, DLKP-I cells grew fastest in this assay, having growth curves almost identical to those of DLKP.

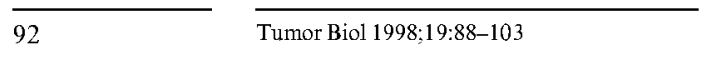




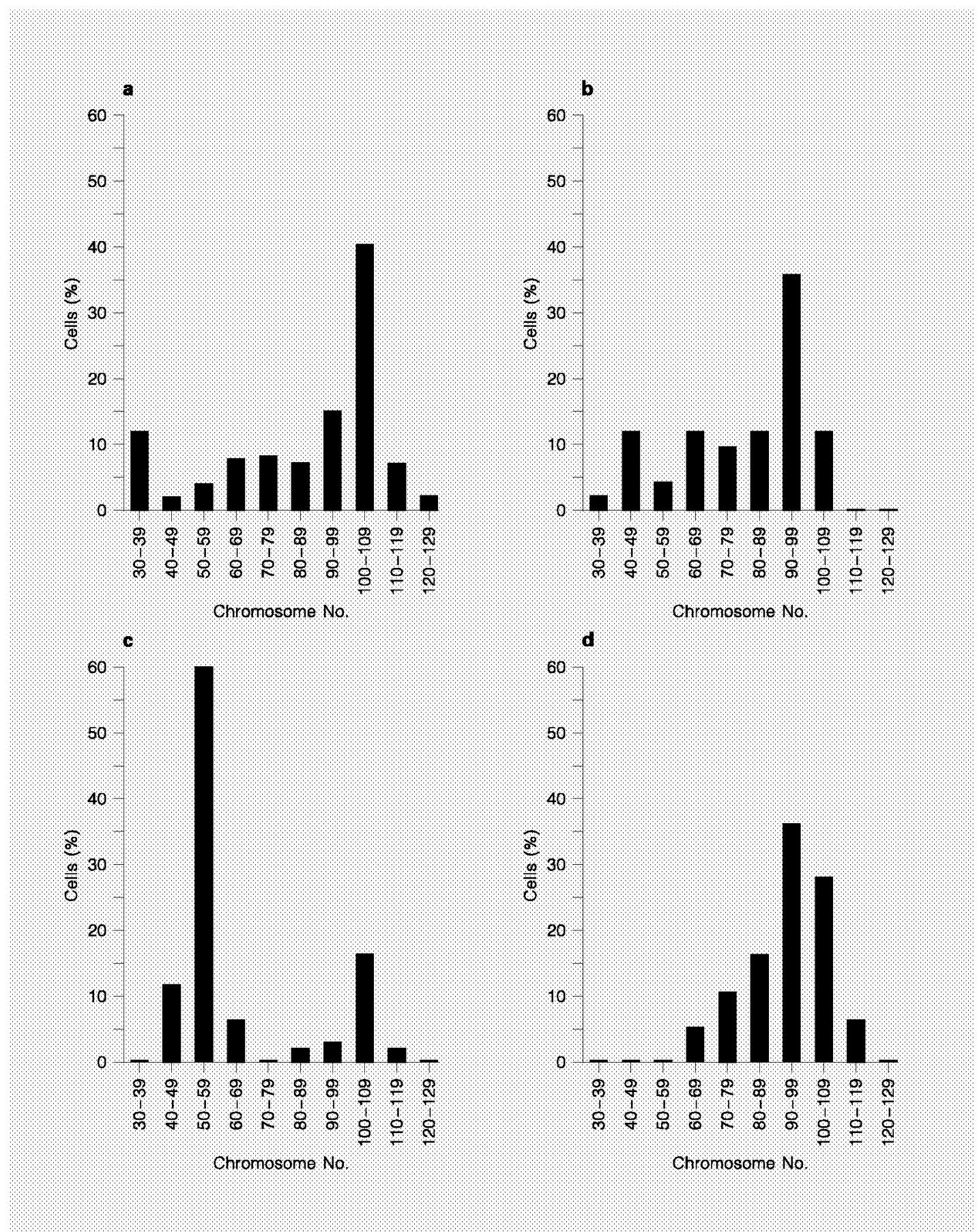

Fig. 3. Range of chromosomal populations determined in DLKP (a), DLKP-I (b), DLKPM (c) and DLKP-SQ (d). In a, the number of metaphase spreads assayed was 75 , and in $\mathbf{b}-\mathbf{d}$, it was 50 .

Clonal Variation in a Lung Cell Line

Tumor Biol 1998;19:88-103

93 


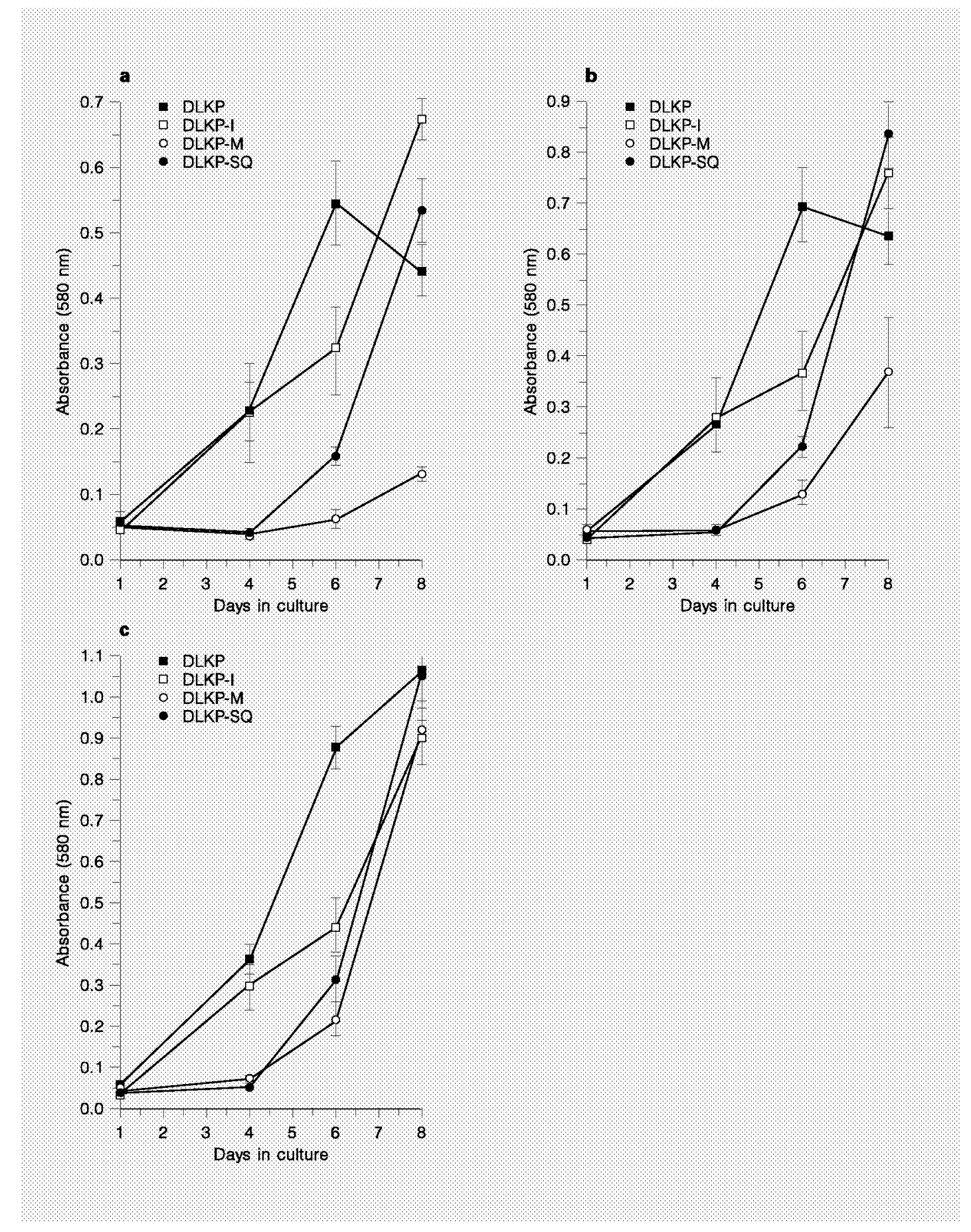

Fig. 4. Growth of DLKP and clones in monolayer culture in the presence of $0 \%$ serum (a), $1 \%$ serum (b) and $5 \%$ serum (c).

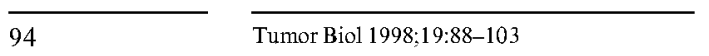




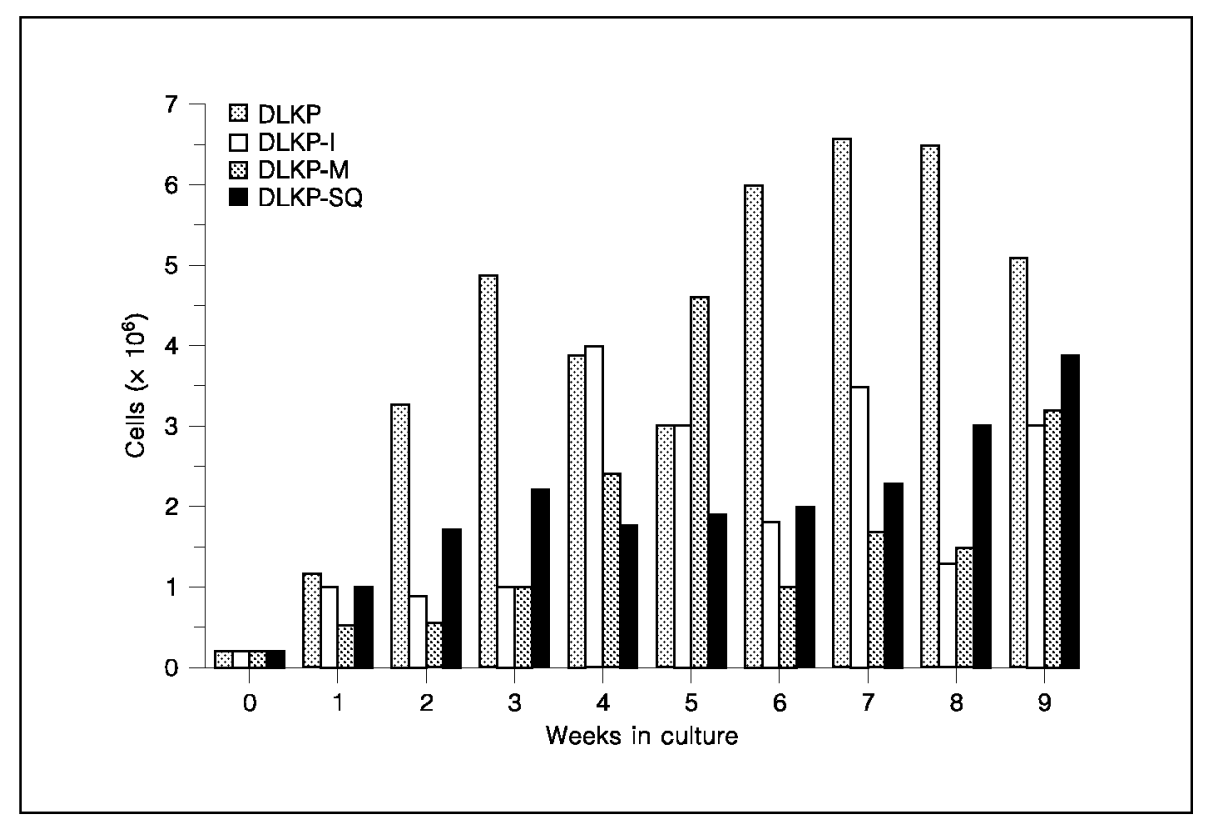

Fig. 5. Growth of DLKP and its clones in spinner flasks. Flasks were initially inoculated with $4 \times 10^{2}$ cells $/ \mathrm{ml}$. Proliferation was determined every 7 days by counting, after which flasks were re-seeded at original inoculating densities.

Table 1. CFE of DLKP and its clones in soft agar

\begin{tabular}{lr}
\hline \multicolumn{1}{c}{} & \multicolumn{1}{c}{ CrE, $\%$} \\
\hline Cell Line & \\
DLKP & $37.58 \pm 7.72$ \\
DLKP-I & $2.45 \pm 2.04$ \\
DLKP-M & $13.08 \pm 0.27$ \\
DLKP-SQ & $26.78 \pm 0.44$ \\
\hline
\end{tabular}

DLKP-SQ and DLKP-M grew relatively poorly in the first few days of culture, appearing to be density dependent, but after this slow start, their growth rates increased also.

The ability of each cell line to form colonies in soft agar was examined. The DLKP cells had the highest CFE ( $38 \%$, table 1$)$. Surprisingly, DLKP-I effectively had no ability to

Clonal Variation in a Lung Cell Line form colonies ( $2.5 \%)$. In contrast, DLKP-SQ had the best CFE of the clones $(27 \%)$, and DLKP-M was intermediate, with a CFE of $13 \%$.

The DLKP cells were also found to be the most efficient of the 4 lines at growing in suspension in spinner flasks (fig. 5). DLKP-SQ were found to grow better than the other 2 clones in this assay, too. All lines grew in aggregates of up to $\sim 1 \mathrm{~mm}$ in diameter. In order to determine whether the lack of substrate adherence and enforced growth in suspension affected the morphology of the cells in any way, aliquots of each cell line were placed in tissue culture flasks after passages 5 and 10 in spinner flasks. After a few days, morphologies of the resulting attached colonies were examined microscopically. The morphologies of cells examined after 5 passages in spinner culture seemed unchanged. 


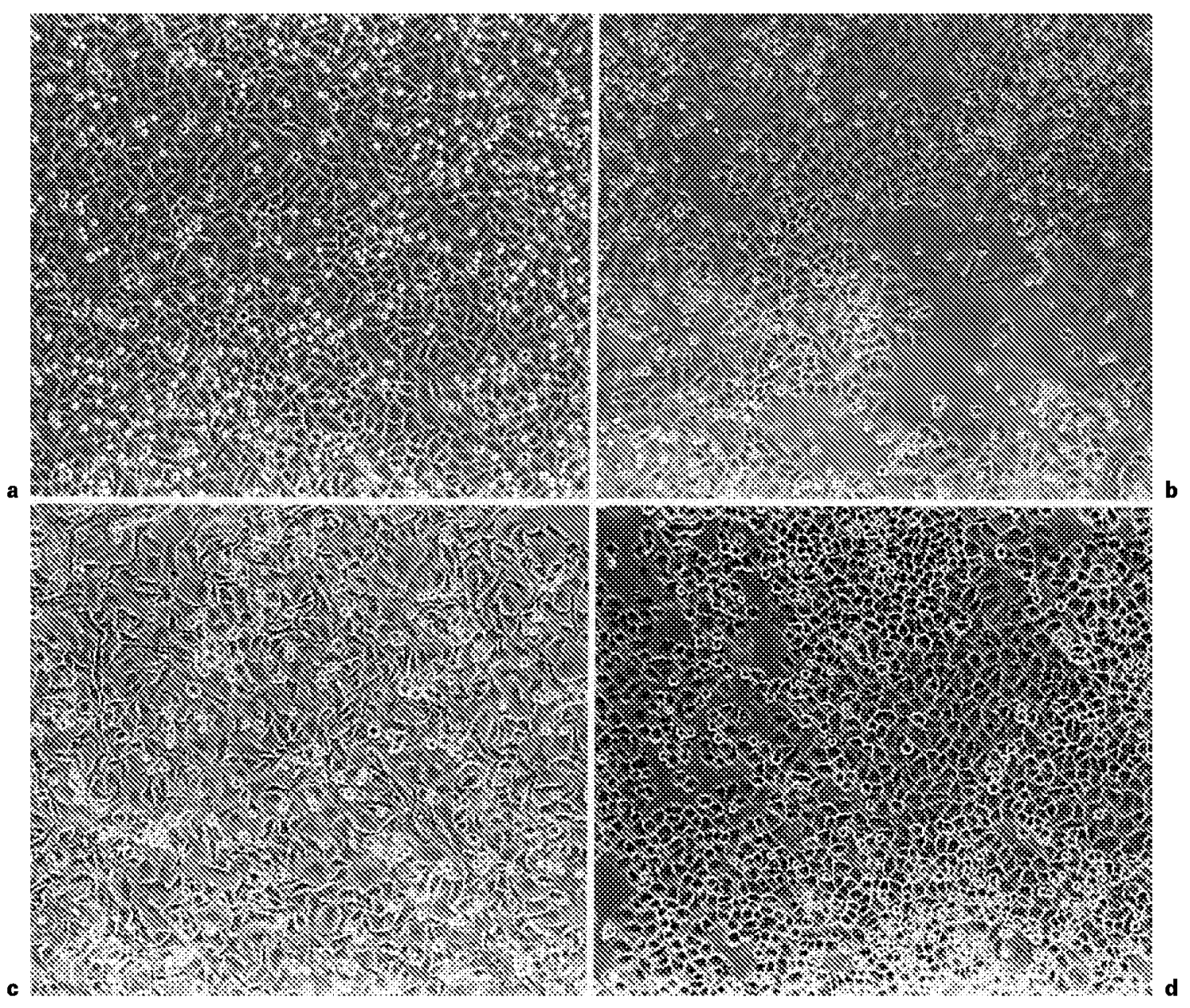

Fig. 6. Appearance of DLKP (a), DLKP-I (b), DLKP-M (c) and DLKP-SQ (d) in monolayer culture in tissue culture flasks after 10 passages growing in suspension in spinner flasks. $\times 100$.

However, after 10 passages, it appeared that the DLKP population had become homogeneous, with all cells having the squamous phenotype of DLKP-SQ (fig. 6). DLKP-SQ itself appeared unchanged, while DLKP-I appeared mixed, having colonies of both its own and the squamous phenotype. DLKP-M also appeared unchanged. It would thus seem that growth of these cells in suspension causes an interconversion towards the squamous phe- notype represented by DLKP-SQ. It is unlikely that selection of a small subpopulation was involved since high numbers of viable cells were grown in all of the cultures.

When cultured in serum-free medium, the numbers of DLKP-SQ and DLKP-M cells failed to increase above seeding-density levels at any stage, regardless of the frequency of medium change or length of time in culture. In contrast, despite an initial slow start,

\footnotetext{
$\overline{96} \overline{\text { Tumor Biol 1998;19:88-103 }}$
} 
Fig. 7. Growth of DLKP and its clones in serum-free medium (DLKP-M and DLKP-SQ failed to proliferate under these conditons) (a), and serum-free medium supplemented with $5 \mu \mathrm{g} / \mathrm{ml}$ fibronectin (b).

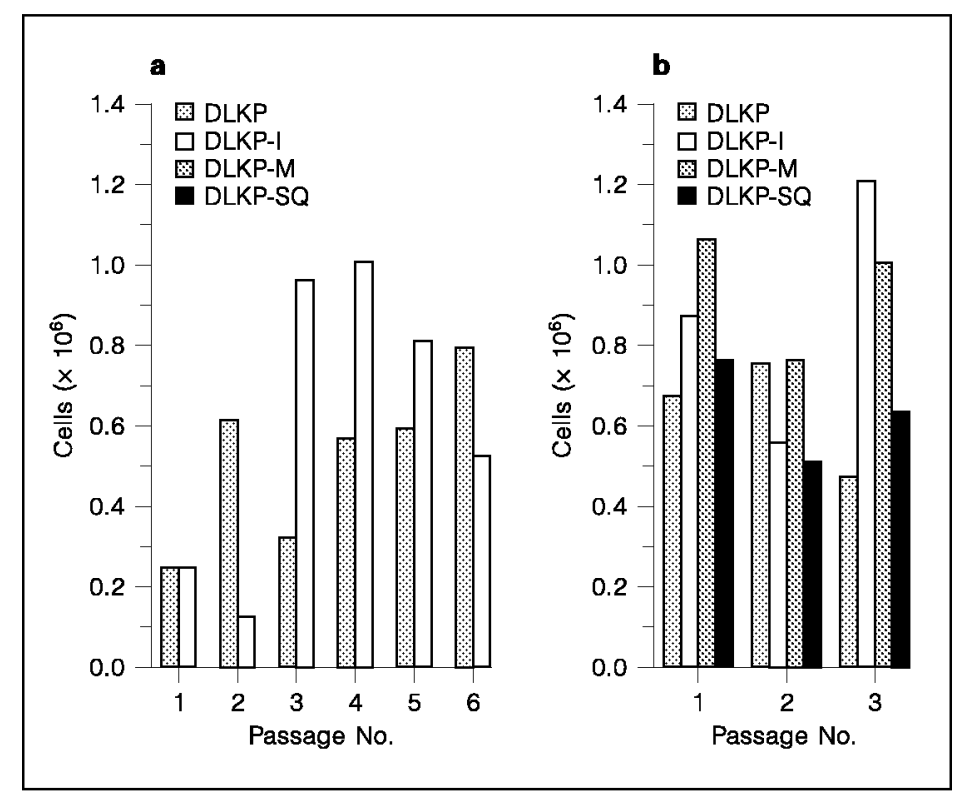

DLKP and DLKP-I grew well under these serum-free conditions and were cultured for up to 10 passages at a time (fig. 7). However, the addition of $5 \mu \mathrm{g} / \mathrm{ml}$ fibronectin to the serum-free medium had a dramatic effect on the proliferation of DLKP-SQ and DLKP-M cells. In the presence of fibronectin, both clones were found to have growth rates similar to those of DLKP and DLKP-I under the same conditions (fig. 7).

\section{Attachment Properties of Clones}

When the rate of attachment of the cells to ECM proteins was examined, it was found that of the 4 lines, DLKP-M adhered more rapidly than the other cell types to fibronectin after $15 \mathrm{~min}$ of incubation and also appeared to have a greater affinity for collagen after $30 \mathrm{~min}$ (fig. 8). In addition, DLKP-M attached more rapidly than the other lines to plastic. The extent of attachment of DLKP-M cells to fibronectin determined after $30 \mathrm{~min}$ was less than that determined after $15 \mathrm{~min}$. In all other cases, attachment was found to increase with time.

\section{Electrical-Resistance Measurements}

The presence of tight junctions in confluent monolayers of DLKP and its clones was determined by measuring the resistance of the monolayers to an electrical current. Increased electrical resistance is indicative of tight junction formation as cells grow tighter together and an epithelial barrier is formed. T84 cells, human colonic cells classically used in these experiments, were used as a positive control. The resistance of these cells increased over 8 days from $80 \Omega$ to over $600 \Omega$ (fig. 9). No increase in resistance was observed in DLKP or its clones, indicating that these cells do not contain tight junctions and are therefore unpolarised epithelial cells.

\section{Determination of Marker Expression}

An extensive immunocytochemical analysis was carried out on DLKP and its 3 clonal 


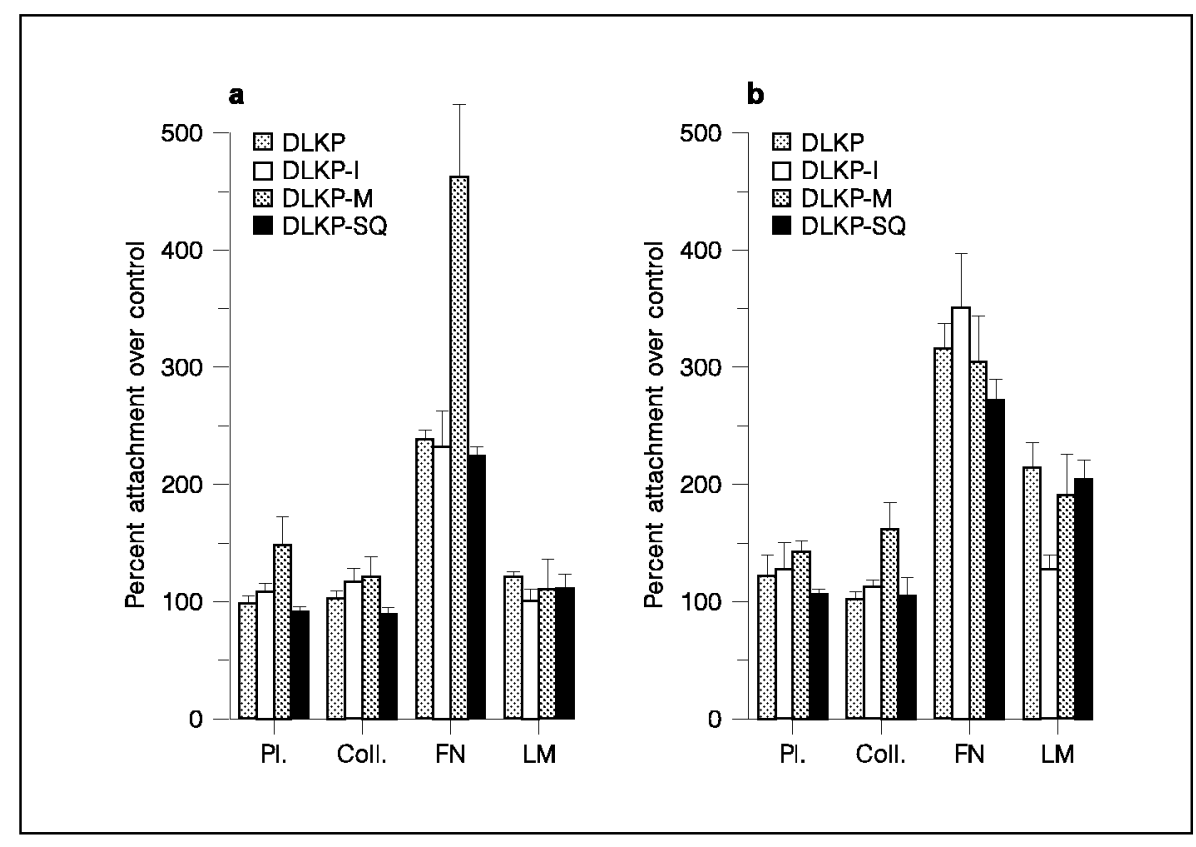

Fig. 8. Attachment of DLKP and its clones to ECM proteins after $15 \mathrm{~min}$ (a) and $30 \mathrm{~min}$ (b). $\mathrm{Pl}=$ plastic; Coll. = collagen type IV; $\mathrm{FN}=$ fibronectin; $\mathrm{LM}=$ laminin.

derivatives in order to characterise the cells and allow comparisons with both tumour and normal lung cell types. The original tumour sample and all 4 DLKP lines derived from it were found to express effectively no cytokeratin intermediate filaments (table 2). Two different antibodies were used: Cam 5.2 which reacts with keratins 8 and 18 and a pan-cytokeratin antibody which detects keratins 1, 4$6,8,10,13,18$ and 19 . With the latter antibody, a very small number of cultured cells $(<1 \%)$ stained positively. Other epithelial markers, epithelial membrane antigen, epithelial-specific antigen, desmosomal protein and desmoplakin, were also absent from the tumour sample, cultured cells or both. Transglutaminase, a marker of squamous differentiation, was not detected, either. These results were unexpected as the tumour had been histologically diagnosed as a poorly differen- tiated squamous cell carcinoma. In contrast, the tumour stained strongly positive for $2 \mathrm{NE}$ markers, NSE and PGP 9.5. DLKP and its clones also reacted positively with NSE and PGP 9.5 antibodies, as well as with an antibody specific for neurofilaments. However, other NE antigens examined were not detectable. The tumour slides were negative for Leu 7 expression, and the cell lines were also negative for neural-cell adhesion molecule, serotonin and chromogranin expression.

While all cultured DLKP cells expressed vimentin, an intermediate filament protein present in cells of mesenchymal origin, the original tumour block contained no vimentinpositive cells. This suggests that vimentin expression was induced as a result of culture conditions rather than being constitutively expressed by DLKP cells. The induction of vimentin expression in non-mesenchymal 
Fig. 9. Electrical resistance exhibited by confluent monolayers of DLKP and each lone.

Table 2. Expression of biological markers in the original tumour biopsy, DLKP (passages 6 and 29) and each of the clones

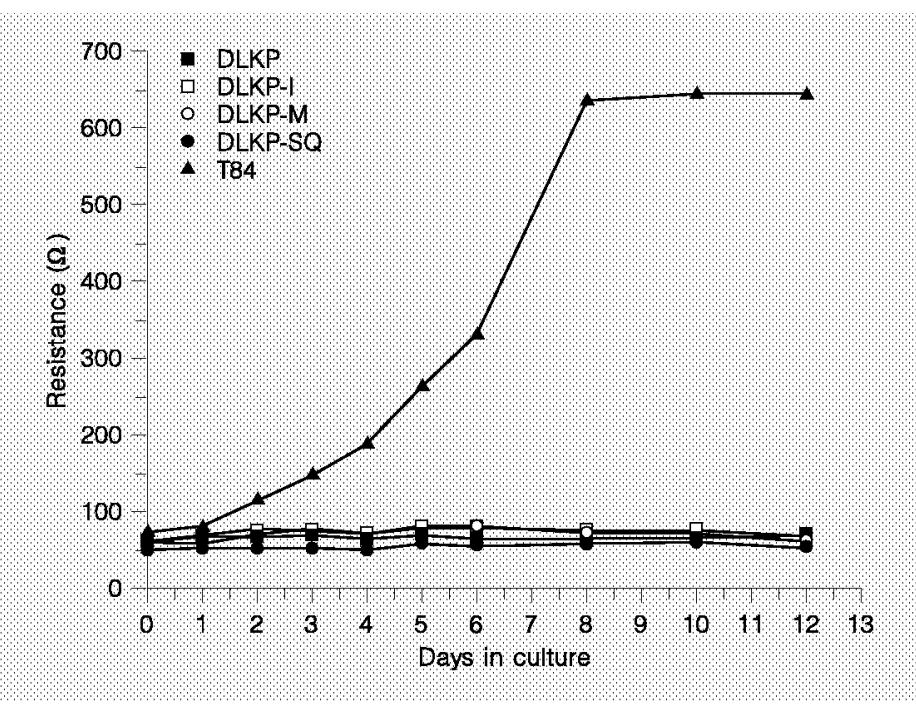

\begin{tabular}{|c|c|c|c|c|c|c|}
\hline Marker & ' & $\begin{array}{l}\mathrm{PL} \\
(\mathrm{P} \sigma)\end{array}$ & $\begin{array}{l}\mathrm{PL} \mathrm{P} \\
(\mathrm{P} 29)\end{array}$ & $\mathrm{DLKP}$ & DLKP M & DLKP SQ \\
\hline Cam 5.2 & Neg. & Neg. & Neg. & Neg. & Neg. & Neg. \\
\hline Cytoker. & n.d. & Neg. & Neg. & Neg. & Neg. & Neg. \\
\hline EMA & Neg. & n.d. & n.d. & n.d. & n.d. & n.d. \\
\hline ESA & n.d. & n.d. & Neg. & Neg. & Neg. & Neg. \\
\hline Desmoplk. & n.d. & n.d. & Neg. & Neg. & Neg. & Neg. \\
\hline Desmo.Pro. & n.d. & n.d. & Neg. & Neg. & Neg. & Neg. \\
\hline Transgl. & n.d. & n.d. & Neg. & Neg. & Neg. & Neg. \\
\hline$\alpha_{2}$-integ. & n.d. & n.d. & Pos. & Pos. & Pos. & Pos. \\
\hline NSE & Pos. & n.d. & Pos. & Pos. & Pos. & Pos. \\
\hline PGP 9.5 & Pos. & Pos. & Pos. & Pos. & Pos. & Pos. \\
\hline Leu 7 & Neg. & n.d. & n.d. & n.d. & n.d. & n.d. \\
\hline NCAM & n.d. & n.d. & Neg. & Neg. & Neg. & Neg. \\
\hline Serotonin & n.d. & n.d. & Neg. & Neg. & Neg. & Neg. \\
\hline Chromogr. & n.d. & n.d. & Neg. & Neg. & Neg. & Neg. \\
\hline Neurofil. & n.d. & n.d. & Pos. & Pos. & Pos. & Pos. \\
\hline GFAP & n.d. & n.d. & Neg. & Neg. & Neg. & Neg. \\
\hline Vimentin & Neg. & n.d. & Pos. & Pos. & Pos. & Pos. \\
\hline Desmin & n.d. & n.d. & Neg. & Neg. & Neg. & Neg. \\
\hline LCA & n.d. & Neg. & Neg. & Neg. & Neg. & Neg. \\
\hline
\end{tabular}

Cytoker. = Cytokeratin; EMA = epithelial membrane antigen; ESA = epithelia-specific antigen; Desmoplk. = desmoplakin; Desmo.Pro. $=$ desmosomal protein; Transgl. $=$ transglutaminase $; \alpha_{2}$-integ. $=\alpha_{2}$-integrin NCAM = neural-cell adhesion molecule; Chromogr. = chromogranin; Neurofil. $=$ neurofilaments; GFAP $=$ glial fibrillary acidic protein; $\mathrm{LCA}=$ lymphocyte common antigen; n.d. = not determined; Neg. = negative; Pos. = positive. 
cells under culture conditions is a frequent occurrence. No cells were positive for glial fibrillary acidic protein or desmin, which are the intermediate filaments present in glial and smooth muscle cells, respectively.

$\alpha_{2}$-Integrin is a membrane glycoprotein involved in cellular binding to ECM proteins. While expression of this protein was detectable in DLKP and each of the clones, DLKP$M$ cells were found to contain higher levels of this integrin than the other clones as determined by the intensity of the immunocytochemical staining (results not shown).

To rule out the possibility that the tumour was in fact lymphatic in origin and not a metastasis from the lung, the presence of lymphocyte common antigen was examined. DLKP and the 3 clones were negative for this marker.

\section{Discussion}

The mechanisms which give rise to karyotypic, morphological and phenotypic tumour heterogeneity are unclear. Genetic instability [26], adaptive evolution [27] and the dominance of a primary lesion by a single clone [28] are believed to be involved in tumour cell progression, but few clonal model systems exist for the study of these processes and for the determination of the physiological conditions which influence them. Khan et al. [1] have reported the isolation of 2 sublines from a classic SCLC cell line which exhibited significant differences in morphology and doubling times from those of the parental line, and Resnicoff et al. [29] report the isolation of subpopulations, including a stemcell-like population, from a breast tumour cell line, but neither cases involved true clonal populations. Another model established by Yao and Rubin [30] examined heterogeneous clonal progression in mouse fibroblasts as determined by morphological and proliferative parameters, illustrating phenotypic conversion, reversal and adaptability of clonal populations in response to growth conditions. This adaptation of tumour subpopulations to growth conditions, similar to our findings with DLKP, is considered part of the carcinogenic process [31] and is likely to be significant in tumour establishment, progression, metastasis and resistance to treatment.

The morphological diversity of the DLKP clones is reflected in their respective chromosome numbers and may indeed be related to them. Burholt et al. [2, 3] observed that the karyotypic evolution of several cultured undifferentiated large cell lung carcinoma cell lines follows an orderly and predictable pattern. The sequence consists of a relatively rapid doubling of the chromosome number, creating a tetraploid population, followed by a gradual loss of chromosomes. It is possible that the 3 DLKP clones, with their distinct chromosomal profiles, represent different stages of a similar process, where a continuous cycle of chromosome doubling and subsequent chromosome loss is occurring. The changes in the balance of gene expression accompanying the fluctuating chromosome populations could result in the diverse, yet interconverting, morphologies observed in the clones.

In addition to morphological and chromosomal differences, growth capacities of the DLKP clones varied greatly under the various growth conditions examined. Of the 3 clones, DLKP-I proliferated the fastest in serum-supplemented and in defined serum-free medium, the latter assay suggesting that this clone is producing autocrine factors which DLKP-SQ and DLKP-M are lacking. Differences in medium requirements were again seen when the addition of a single factor, fibronectin, was found to be capable of stimulating growth of DLKP-SQ and DLKP-M to

$100 \quad$ Tumor Biol 1998;19:88-103

McBride/Meleady/Baird/Dinsdale/

Clynes 
levels similar to DLKP-I, while no such growth increase was seen in DLKP-I itself. In contrast, DLKP-SQ had the best CFE in soft agar and also grew best when cultured in suspension in spinner flasks, again illustrating differences in the growth potentials of the 3 clonal populations.

In our studies, we observed that the clonal subpopulations derived from DLKP are capable of morphological conversions depending on growth conditions. It seems that the DLKP-I cells may be an intermediate phenotype between those of DLKP-SQ and DLKP$\mathrm{M}$ or possibly a stemcell-like population which can give rise to either of the other 2 phenotypes, depending on growth conditions. Growth in suspension in spinner flasks which allows the formation of 3-dimensional colonies led to the mixed DLKP population becoming homogeneously squamous in appearance (similar in morphology to DLKP-SQ, which was the clone which grew best under these conditions), and the DLKP-I phenotype also changed towards a squamous morphology. The original tumour biopsy was also histologically diagnosed as a poorly differentiated squamous carcinoma. It thus appears that the squamous phenotype exhibited by these cells may be suitable for their growth in the absence of a surface to colonise. The apparent ability of DLKP-SQ to revert to the DLKP-I morphology in culture reflects the plasticity of tumour cells which allows them to survive and to adapt to their environment.

The interconversion between DLKP-I and DLKP-M may also reflect this plasticity. DLKP-M has several features seen in mesenchymal-type cells. For example, the DLKP-M cells do not tend to grow in a monolayer, but as scattered cells with a fibroblast-like morphology and with processes which often appear to extend over each other. Even in parental DLKP cultures, they do not appear to form cell-cell contacts. Metastatic cells must attach to the basement membrane, where they enzymatically work their way into the vascular system and into circulation [32]. Of the 4 lines, the DLKP-M cells were found to attach most rapidly to the ECM proteins, collagen and fibronectin. In the case of fibronectin, the cells initially appeared to attach rapidly (15 min), but then seemed to detach again. The adhesion properties of metastasising cells are complex [33], and their interactions with ECM proteins must allow for substrate adhesion, but also motility. The ECM attachment and detachment kinetics of DLKP-M cells may reflect these interactions. Additional work must be carried out to determine the metastatic potential of the DLKP-M cells and whether they do represent a metastatic phenotype, and further study of the DLKP-I/ DLKP-M interconversion should prove beneficial to our understanding of tumour progression.

Extensive characterisation work which included physiological and biological studies was carried out here in order to identify as accurately as possible the cell types, both normal and tumour, which DLKP and its clones may represent in vivo. When examined by transmission electron microscopy (carried out by Dr. David Dinsdale, MRC Toxicology Unit, University of Leicester, UK), neither the clones nor their parental cells, DLKP, exhibited any distinguishing ultrastructural characteristics such as dense core granules or lamellar bodies, which are present in NE and secretory cells, respectively, suggesting an overall lack of differentiation (results not shown). In addition, tight junctions, which are present in normal epithelial cells, do not appear to be present in any DLKP cells. The immunological studies concurred with these observations, with the failure to detect any epithelial markers examined. Therefore, DLKP and its clones may represent a poorly differentiated stemcell-like lung epithelial li- 
neage which has potential for both proliferation and differentiation. In the mature lung, no distinct stem cell lineage has yet been identified, but at least 3 cell types, Clara, Type II and basal cells, are capable of non-terminal division and differentiation. Of these 3 cell types, DLKP cells most closely resemble basal cells with regard to their lack of differentiation features.

The range of biological markers expressed (and sometimes more significantly not expressed) in the DLKP cells and the tumour from which they were derived is consistent with that of variant SCLC cells or NSCLCNE. The absence of cytokeratin intermediate filaments coupled with the presence of neurofilaments is typical of SCLC-V [34]. Similarly, the continued expression of PGP 9.5 and in particular NSE in the absence of several other NE markers such as neural-cell adhesion molecule and elements of the APUD system such as dense core granules, chromogranin A and serotonin (5-HT) is another criterion for SCLC-V classification [15]. In addition, conversion to a NSCLC-like morphology is often evident in SCLC-V cells [15], and again, consistent with this, the DLKP cells grow mainly attached, with a small proportion $(<5 \%)$ growing in suspension. NE cells are the first mature cell type to appear in the developing lung. NSE immunoreactivity is one of the earliest NE markers observed in these cells, appearing before most neuropeptides can be detected [35]. Therefore, the presence of NSE positivity in cells which do not contain neuropeptides or APUD elements as is the case with DLKP, may indicate that such cells are at a very early stage of differentiation and can theoretically progress towards several distinct phenotypes. Maintaining this plasticity is of obvious benefit to tumour cells as they remain uncommitted to any single differentiation pathway and so are free to adapt to a changing environment. DLKP and its clonal variants may prove to be a useful model for the study of tumorigenic progression. In particular the possible identification of DLKP-I as a stemcell-like population may give insight into both normal and aberrant developmental processes in the lung.

\section{References}

1 Khan MZ, Freshney RI, Murray AMB, Merry S, Plumb JA, McNicol AM: Identification and characterisation in vitro of cells with a nonSCLC cell-like phenotype derived from a continuous SCLC cell line. Anticancer Res 1991;11:16871696.

2 Burholt DR, Shackney SE, Ketterer DM, Pollice AA, Smith CA, Brown KA, Giles HR, Schepart BS: Karyotypic evolution of a human undifferentiated large cell carcinoma of the lung in tissue culture. Cancer Res 1989;49:3355-3361.

3 Burholt DR, Shackney SE, Ketterer DM, Pollice AA, Smith CA, Giles HR, Hartsock RJ: Tetraploidization, chromosome loss and the production of structural chromosomal abnormalities in a human lung adenocarcinoma cell line. Proc Am Assoc Cancer Res 1989;30:37.

4 Terasaki T, Kameya T, Nakajima T, Tsumuraya M, Shimosato Y, Kato K, Ichinose H, Nagatsu T, Hasegawa $\mathrm{T}$ : Interconversion of biological characteristics of small cell lung cancer depending on culture conditions. Gann 1984;75:1089-1099.

5 Young MR, Newby M, Meunier J: Relationships between morphology, dissemination, migration, and prostaglandin $\mathrm{E}_{2}$ secretion by cloned variants of Lewis lung carcinoma. Cancer Res 1985;45:3918-3923.
6 Moss F, Bobrow L, Happerfield L, Souhami R, Beverly P: Differential expression of antigens by human small cell lung carcinoma in sections of tumour and in serous effusions. Br J Cancer 1991;63(suppl XIV): 54-55.

7 Mooi WJ, Dingemans KP, Wagenaar S, Hart AAM, Wagenvoort CA Ultrastructural heterogeneity of lung carcinomas: Representativity of samples for electron microscopy in tumor classification. Hum Pathol 1990;21:1227-1234

8 Inayama Y, Hook GE, Brody AR Jetten AM, Gray T, Mahler J, Nettesheim $P$ : In vitro and in vivo growth and differentiation of clones of tracheal basal cells. Am J Pathol 1989 134:539-549.

$\overline{102} \quad \overline{\text { Tumor Biol 1998;19:88-103 }}$

McBride/Meleady/Baird/Dinsdale/

Clynes 
9 World Health Organisation: The World Health Organisation histological typing of lung tumours. Am J Clin Pathol 1982;77:123-136.

10 Gazdar AF, Helman LJ, Israel MA, Russell EK, Linnoila RI, Mulshine JL, Schuller HM, Park J: Expression of neuroendocrine cell markers $L$ dopa decarboxylase, chromogranin $\mathrm{A}$, and dense core granules in human tumors of endocrine and nonendocrine origin. Cancer Res 1988; 48:4078-4082.

11 Gazdar AF, Zweig MH, Carney DN, Van Steirteghen AC, Baylin SB, Minna JD: Levels of creatine kinase and its BB isoenzyme in lung cancer specimens and cultures. Cancer Res 1981;41:2773-2777.

12 Said JW, Vimadalal S, Nash G, Shintaku I, Heusser RC, Sassoon AF, Lloyd RV: Immunoreactive neuron-specific enolase, bombesin and chromogranin as markers for neuroendocrine lung tumors. Hum Pathol 1985;16:764-768.

13 Bergh J, Esscher T, Steinholtz L, Nilsson K, Pahlman S: Immunocytochemical demonstration of neuron-specific enolase (NSE) in human lung cancers. Am J Clin Pathol 1985;84:1-7

14 Bepler G, Jaques G, Neumann K Aumuller G, Gropp C, Havemann $\mathrm{K}$ : Establishment, growth properties, and morphological characteristics of permanent human small cell lung cancer cell lines. J Cancer Res Clin Oncol 1987:11:31-40.

15 Carney DN, Gazdar AF, Bepler G, Guccion JG, Marangos PJ, Moody TW, Zweig MH, Minna JD: Establishment and identification of small cell lung cancer cell lines having classic and variant features. Cancer Res 1985;45:2913-2923.

16 Watanabe H, Takahashi T, Ueda R, Utsumi KR, Sato T, Ariyoshi Y, Ota K, Obata Y, Takahashi T: Antigenic phenotype and biological characteristics of two distinct sublines derived from a small cell lung carcinoma cell line. Cancer Res 1988;48: 2544--2549

17 Burnett HE, Zakhour HD, Walker C: Neuroendocrine and epithelial markers in diagnostic bronchial lung cancer biopsy specimens. Eur J Cancer 1992;28A:853-855.
18 Kasi K, Kameya T, Kawakubo Y, Sato Y, Wada C, Itoh H: Pulmonary large cell carcinoma expressing neuroendocrine markers: The morphological, biological, and neuroendocrine features of their cell lines and surgical cases. Jpn J Cancer Res 1992;83:1002-1010.

19 Sundaresan V, Reeve JG, Stenning S, Stewart S, Bleehan NM: Neuroendocrine differentiation and clinical behaviour in non-small cell lung tumours. Br J Cancer 1991;64:333338.

20 Skov BG, Sorensen JB, Hirsch FR, Larsson LI, Hansen HH: Prognostic impact of histologic demonstration of chromogranin A and neuron specific enolase in pulmonary adenocarcinoma. Ann Oncol 1991;2:355360.

21 Carney DN, Mitchell JB, Kinsella TJ: In vitro radiation and chemotherapy sensitivity of established cell lines of human small cell lung cancer and its large cell morphological variants. Cancer Res 1983;43: 2806-2811

22 Gazdar AF, Carney DN, Guccion JC, Baylin SB: Small cell carcinoma of the lung: Cellular origin and relationship to other pulmonary tumors; in Greco FA, Oldham RK, Bunn PA (eds): Small Cell Lung Cancer. New York, Grune and Stratton, 1981, pp 145-175.

23 Law E, Gilvarry U, Lynch V, Gregory B, Grant G, Clynes M: Cytogenetic comparison of two poorly differentiated human lung squamous cell carcinoma lines. Cancer Genet Cytogenet 1992;59:111-118.

24 Martin A, Clynes M: Comparison of 5 microplate colorimetric assays for in vitro cytotoxicity testing and cell proliferation assays. Cytotechnology 1993;11:49-58.

25 Mendiaz E, Mamounas M, Moffett J, Englesberg E: A defined medium for and the effect of insulin on the growth, amino acid transport and morphology of Chinese hamster ovary cells, CHO-K1 (CCL 61) and the isolation of insulin 'independent' mutants. In Vitro Cell Dev Biol 1 986:22:66-74
26 Bailly M, Bertrand S, Dore JF: Increased spontaneous mutation rates and prevalence of karyotype abnormalities in highly metastatic human melanoma cell lines. Melanoma Res 1993;3:51-61

27 Rubin H: Adaptive evolution of degrees and kinds of neoplastic transformation in cell culture. Proc Nat Acad Sci USA 1992;89:977-981.

28 Theodorescuan D, Cornil I, Sheehan C, Man S, Kerbel RS: Dominance of metastatically competent cells in primary murine breast neoplasms is necessary for distant metastatic spread. Int J Cancer 1991;47: 118-123.

29 Resnicoff M, Medrano EE, Podhaj cer OL, Bravo AI, Bover L, Mordoh $\mathrm{J}$ : Subpopulations of MCF7 cells separated by Percoll gradient centrifugation: A model to analyze the heterogeneity of human breast cancer. Proc Natl Acad Sci USA 1987 84:7295-7299.

30 Yao A, Rubin H: Automatic enumeration and characterization of heterogenous clonal progression in cell transformation. Proc Natl Acad Sci USA 1993;90: 10524-10528.

31 Farber E, Rubin H: Cellular adaptation in the origin and development of cancer. Cancer Res 1991;51: 2751-2761.

32 Liotta LA, Steeg PS, Stetler-Stevenson WG: Cancer metastasis and angiogenesis: An imbalance of positive and negative regulation. Cell 1991 64:327-336.

33 Birchmeier W, Behrens J: Cadherin expression in carcinomas: Role in the formation of cell junctions and the prevention of invasiveness. Biochim Biophys Acta 1994;1198:1126.

34 Broers JLV, Carney DN, de Ley L, Vooijs GP, Ramaekers FCS: Differential expression of intermediate filament proteins distinguishes classic from variant small-cell lung cance cell lines. Proc Natl Acad Sci USA 1985;82:4409-4413.

35 Cutz E: Neuroendocrine cells of the lung. An overview of morphologic characteristics and development Exp Lung Res 1982;3:185-208. 